\title{
Pierre-W. Boudreault
}

Sociologue, professeur au départgement des sciences humaines, UQAC

(1991)

\section{"Anse Saint-Jean et la lutte pour la survie de l'éco-système"}

Un document produit en version numérique par Jean-Marie Tremblay, bénévole, professeur de sociologie au Cégep de Chicoutimi

Courriel:jmt_sociologue@,videotron.ca

Site web: http://www.uqac.ca/jmt-sociologue/

Dans le cadre de la collection: "Les classiques des sciences sociales"

Site web: http://www.uqac.ca/Classiques_des_sciences_sociales/

Une collection développée en collaboration avec la Bibliothèque

Paul-Émile-Boulet de l'Université du Québec à Chicoutimi

Site web: http://bibliotheque.uqac.uquebec.ca/index.htm 
Cette édition électronique a été réalisée par Jean-Marie Tremblay, bénévole, professeur de sociologie au Cégep de Chicoutimi à partir de l'article de :

Pierre-W. Boudreault

[sociologue, professeur au département des sciences humaines, Université du Québec à Chicoutimi.]

“Anse-Saint-Jean et la lutte pour la survie de l'éco-système” (1991)

Un article publié dans la revue Possibles. vol. 15, no 1, hiver 1991, pp. 147$163 "$.

[Autorisation formelle accordée par l'auteur le 17 mai 2005.]

89 Courriel : Pierre-W_Boudreault@uqac.ca

Polices de caractères utilisée :

Pour le texte: Times, 12 points.

Pour les citations : Times 10 points.

Pour les notes de bas de page : Times, 10 points.

Édition électronique réalisée avec le traitement de textes Microsoft Word 2004 pour Macintosh.

Mise en page sur papier format

LETTRE (US letter), 8.5' x 11'')

Édition complétée le 19 juillet 2005 à Chicoutimi, Ville de Saguenay, province de Québec.

\section{Fait avec}


Pierre-W. Boudreault

[sociologue, professeur au département des sciences humaines, Université du Québec à Chicoutimi.]

\section{"Anse-Saint-Jean et la lutte pour la survie de l'éco-système" (1991)}

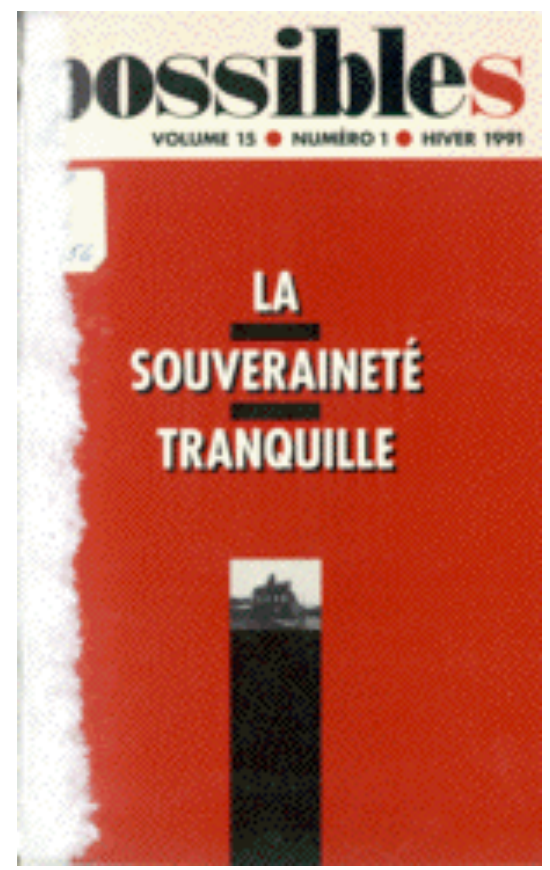

Un article publié dans la revue Possibles. vol. 15, no 1, hiver 1991, pp. 147-163”. 


\section{Table des matières}

Introduction

1. C'est le jeu qui forme l'acteur qui, à son tour, se fait prendre au jeu !

2. Comment un projet devient un dossier : la Corporation

3. La révolte sociale contre la façade administrative

4. La scie mécanique et le goupillon contre les fantômes de la société industrielle 


\section{Introduction}

«Le sens est tout entier dans la manière, la manière est, dans chaque aspect technique et tonal, partie intégrante du sens » (George Steiner, dans Martin Heidegger, Paris : Champs/Flammarion, 1981. p. 103).

Le choix de l'apôtre Saint-Jean, comme patron et protecteur de la petite colonie née en 1838 ne peut pas être sans signification. Saint-Jean n'est-il pas en effet l'apôtre de l'amour dans la religion chrétienne ? N'est-il pas la figure de la réconciliation et l'expression de l'harmonie retrouvée entre des forces opposées ? Il faut avoir vu le relief contrasté à cette hauteur du Saguenay pour comprendre qu'au fond les pionniers qui s'attaquent au fjord affrontent les forces de la nature qui ici atteignaient leurs limites. Les plus hauts sommets côtoient les fosses abyssales. Les cimes qui pointent le toit de la région voisinent le noir des plus profondes eaux. La nature se dédouble comme pour donner l'impression de vivre un combat millénaire entre gladiateurs où l'homme n'a plus aucun pouvoir... naturel.

Les premiers individus à vouloir dompter cette nature, ces propagateurspartageurs du progrès, ces héritiers de la conception cartésienne de la nature, tout chrétiens qu'ils sont, contre la force déchaînée d'Apollon invoquent celui qui rassemble dans la communion, celui qui fusionne dans la communauté pour former équipe face à cette nature hostile. Saint-Jean est ici interpellé comme le prêtre intercède celui qui symbolise les forces du bien capable d'exorciser les 
démons qui visiblement possèdent ce coin de la planète. Il faut ajouter que SaintJean, dont la fête est célébrée au solstice d'été, symbolise traditionnellement la réconciliation et le moment de la célébration publique de la fusion des forces de la vie.

Saint-Jean, Dionysos chrétien, est celui qui, dans : « l'ivresse » (...) « abolit la subjectivité de l'individu jusqu'à ce qu'il s'oublie complètement »... parce que transporté par Apollon, dieu des énergies, du rêve, de l'imagination, l'homme s'aperçoit qu'il s'était laissé emporter par les rêves, les apparences. Alors pris d'« effroi », s'apercevant que les phénomènes l'avaient égarés et que le principe de causalité faisait défaut», l'homme se retrouve dans «les frissons de l'ivresse ». Frédéric Nietzsche écrit encore : "C'est par des chants et des danses que l'homme se manifeste comme mesure d'une collectivité qui le dépasse (...). Ses gestes montrent qu'il est ensorcelé. Maintenant les bêtes parlent, la terre donne du lait et du miel, et en l'homme aussi quelque chose de surnaturel s'exprime. Il se sent dieu ; porté au-dessus de lui-même, il foule le sol, extasié, comme dans son rêve il a vu faire aux dieux. L'homme n'est pas artiste, il est devenu oeuvre d'art : ce qui dans la nature est créateur d'art se révèle ici dans les frissons de l'ivresse pour la profonde délectation de l'être originel $»^{1}$

$$
* * *
$$

Village de 1371 personnes en 1986, Anse-Saint-Jean décide de faire l'histoire. À partir de cette année 1986, les séquestrés de la politique de régionalisation du Québec sortent de leur huis clos. L'enfer, ce ne sera plus l'autre nous, pourrait-on dire. À travers une lutte de citoyens, un nouveau réenchantement du monde, une nouvelle magie jaillit on ne sait trop de la force tellurique qui jadis, façonna cette scandinavie québécoise ou de la puissance surhumaine de ces hommes et de ces femmes héritiers de leurs conquérants pour le moins insolents qui, en 1838, débarquèrent sur les rives de ce havre, amphithéâtre naturel le long d'un fiord, témoin des tumultes d'une genèse que les impétueux colonisateurs de l'Anse ont consacrée à Saint-Jean.

Dans ce texte, nous voudrions saisir la signification des gestes posés par les citoyens de l'Anse-Saint-Jean, gestes qui ont conduit à l'obtention d'un centre de ski alpin du Mont Édouard. Pour cela, il faut savoir quels étaient les référents symboliques utilisés au cours des luttes sociales menées par les citoyens. De quel univers symbolique les luttes des citoyens sont-elles révélatrices?

Frédéric Nietzsche, La naissance de la tragédie, Paris, Gonthier, 1964, pp. 21-22. 
La bataille menée pendant quatre ans par les citoyens se termine dans l'allégresse. L'heureux dénouement d'une très longue lutte n'est certes pas étrangère à la nature sociale de ses acteurs. Or, la force de changement ne reposet-elle pas sur le fait que ces acteurs sociaux ont su se réapproprier la zone d'incertitude de l'action sociale de manière telle que les agents économiques et politiques en place dans les institutions vouées au développement régional seraient tombés dans une méta-logique totalement étrangère à la société que nous appellerons post-moderne?

La bataille conduite pendant quatre ans par les citoyens de l'Anse-Saint-Jean ne peut être saisie sans aborder l'univers culturel auquel il est fait référence à chaque épisode de son déroulement. Les références à la famille prennent des dimensions pour le moins méta-sociales. Les références à la foi chrétienne et aux écritures saintes sont manifestées non seulement par le rôle joué par le curé, mais dans l'ensemble des conduites collectives des paroissiens. Les acteurs principaux sont les bûcherons de la paroisse. Mais est-ce encore des bûcherons au moment où on abat les arbres des futures pistes de ski ? Ne seraient-ils pas déjà des officiers responsables de la gestion d'un éco-système dans lequel s'insèrent des opérateurs et des techniciens d'équipement récréo-touristique au nombre duquel figure un centre de ski alpin, un complexe d'hébergement touristique, un centre d'interprétation du patrimoine, une marina, un centre équestre, etc.

Au fond, si les initiatives socio-économiques des citoyens ont dû s'imposer à travers une bataille dont le mouvement a duré 4 années, ce n'est pas précisément dû au fait que depuis le début des années 1970, le Québec, en se dotant d'une politique de régionalisation, a répandu sur son territoire historique des appareils administratifs dont les agents sont des bureaucrates sans aucune obligation ni responsabilité directe devant la population. En l'absence de démocratie appropriée, les fonctionnaires s'apparentent à une nomenclature et à leur corps défendant offrent une résistance à tous les changements qui peuvent survenir dans la société. Issues d'une conception sociale aujourd'hui dépassée, les institutions dites de « développement régional » sont non seulement des dinosaures à traîner mais des officines dysfonctionnelles par rapport au développement de la population québécoise. 


\section{C'est le jeu qui forme l'acteur qui, à son tour, se fait prendre au jeu !}

$\underline{\text { Retour à la table des matières }}$

La Jeune chambre de l'Anse-Saint-Jean organise en 1986, un colloque dont le but est de réunir ceux et celles qui sont en mesure de formuler des alternatives originales en matière de relance économique de la communauté en voie de devenir une « réserve de personnes âgées ». Depuis dix ans, la population a chuté de 8,78\%, passant de 1503 en 1976 à 1371 personnes en 1986. Le témoignage du curé de la paroisse résume assez l'état de situation démographique et donne l'heure juste sur l'état d'esprit de la communauté.

En fait, c'était pour nous autres, une lutte pour notre survie. Si on n'avait pas ça (un nouveau projet moteur de relance économique), qu'est-ce que l'Anse-SaintJean serait devenu ? Il serait devenu un village fantôme (...) sur les dix mariages, en 1989, pas un seul couple s'est établi (...) nous avons de plus perdu deux familles, une trentaine de maisons sont à vendre (...) les jeunes étudient la possibilité de sortir d'ici et ce, dès qu'ils sont rendus au secondaire sinon au CEGEP...) alors, les touristes viendraient voir ici un parc de personnes âgées, une réserve de personnes âgées. (Témoignage recueilli le 28 mai 1990)

C'est au moment de ce colloque que l'imagination et la culture sont mises à l'épreuve. Alors que l'on suggère l'urgence de mettre sur pied des usines de transformation du bois (vg. cure-dents, hockey, etc.), A. B., fils d'une lignée de marchands établis à l'Anse depuis le début de la colonisation, avance l'idée de créer un centre de ski alpin intégré au concept récréo-touristique quatre saisons qui est en train de se mettre en place à la périphérie de l'actuel Parc du basSaguenay. Anse-Saint-Jean sis au pied d'un massif montagneux, village en ruban, coincé entre les rives du fjord et la montagne qui découpent des vallées encaissées où s'entassent jardins et prés, mettrait en valeur son éco-système là où des générations de bûcherons « en ont arraché » pour extraire le bois d'oeuvre, puis le bois de pulpe et de chauffage.

Dans les jours qui suivent, le journal Le Quotidien, qui diffuse sur l'ensemble de la région, publiait en page couverture la nouvelle que l'Anse-Saint-Jean aurait son centre de ski alpin. D'abord relativement bien accueillie lors du colloque, 
ensuite objet de réflexion de la part des membres du conseil municipal plus préoccupés par leur plan d'urbanisme que par l'un ou l'autre des projets, l'idée emportait graduellement l'opinion orientée vers une nouvelle vision de leur économie traditionnellement centrée sur l'exploitation de leur forêt.

Dans les semaines qui suivent l'annonce dans le journal régional du proie de réalisation d'un centre de ski alpin, une assemblée populaire manifeste son enthousiasme. C'est là que les leaders ont, pour la première fois, l'occasion d'expliquer la nature et les objectifs du projet. À la surprise des élus municipaux qui avaient rejeté l'idée d'un financement et d'une réunion sur cette question, les organisateurs recueilleront l'assentiment général de la population. L'appui populaire est massif en nombre et en acte puisque spontanément on recueillera plus de 25000 \$ pour le financement d'une étude de préfaisabilité requise pour l'obtention des subventions accordées dans le cadre des accords de développement de la zone périphérique du parc du Bas-Saguenay (Le Bas-Saguenay est la zone riveraine du Saguenay qui comprend la partie la plus montagneuse fu fjord et où végètent de petits villages historiques sis dans des amphithéâtres naturels résultats d'affaissements de terrain le long de lignes de faille).

Dans cette assemblée, sous la direction de leaders naturels, un nouveau lien réunit les familles. "Avant de mourir, il faut bien faire un dernier souffle (...) le monde intéressé à vivre à l'Anse-Saint-Jean, à se prendre en main, à survivre, à penser à nos enfants puis à nos jeunes, donnez-nous de l'argent pour prouver que le mont Édouard peut être faisable (...). Alors, le premier à se lever Jans la salle a été M. Louis-Joseph Houde, 80 ans, met 100 \$ sur la table », affirme mon interlocuteur.

Selon le témoignage recueilli auprès de ce dynamique fils de marchand général, cette idée n'était pas le fruit du hasard. Pour que la graine se transforme en germe, il faut une terre d'accueil. Le colloque local fut, dans ce cas, le terrain fertile à la germination qui, dans la société, s'appelle la communication. L'idée fit son nid dans l'opinion. Le bouillon de culture ayant été soigneusement entretenu in vitro par une bande d'amis sportifs qui se demandaient depuis plusieurs années s'ils ne pouvaient pas profiter de leurs « sacrées » montagnes au lieu d'aller skier au Mont Grand-Fonds (Charlevoix) ou au Valinouët (180 km au nord de l'AnseSaint-Jean). Bref, comment se fait-il que la plus haute montagne du Saguenay ne profite-t-elle pas aux skieurs(ses) de tout le territoire?

Une véritable conversion était en train de bâtir son nid. Et si le relief escarpé des montagnes n'était plus la «misère noire » essuyée par les bûcheux pendant plus de 150 ans. Et si la montagne existait non pas pour ce qu'elle recèle mais pour ce qu'elle est, non pas pour ce qu'elle cache mais pour ce qu'elle offre. Relief 
rébarbatif à la coupe forestière qui en a fait «arracher » plus d'un, les fils et les filles des bûcheux à la solde de compagnies extérieures au village et à la région semblaient être en train de changer leur philosophie de la nature et leur relation à la nature, bref, à changer leur philosophie de l'économie.

Pour ce faire, encore fallait-il passer à travers un long processus, re-créer en quelque sorte une nouvelle société. Encore fallait-il que les différentes familles enfermées dans leur fierté et coupées les unes des autres par leur rivalité ancestrale, au-delà des clivages et segmentations séculières, forment une coalescence. Il fallait qu'un adversaire commun se dresse sur leur route pour que se forge une identité au-delà des divergences. Le village scotomisé en trois segments (la zone du port, le village-faubourg, le coin) devait trouver un adversaire assez puissant pour neutraliser les forces endémiques de division. Les divisions entre les familles avaient été, à l'époque de l'industrie forestière, les conditions essentielles et déterminantes pour motiver les hommes et les femmes à surmonter l'ingratitude de la montagne. Devant l'inéluctable, la raison succombe et dans le bruit des salles de réunions populaires, une émotion commune relègue les querelles, efface les susceptibilités, établit un nouveau courant et tisse un nouveau lien.

\section{Comment un projet devient un dossier : la Corporation}

$\underline{\text { Retour à la table des matières }}$

Huit mois plus tard, la firme retenue pour l'étude, conclut à la faisabilité du projet. Les responsables, forts de cette expertise, demandent une rencontre intermunicipale des villages du Bas-Saguenay et du préfet de la MRC afin de présenter le rapport.

C'est là, poursuit mon interlocuteur, que notre petite gang se fait complètement tasser de la route. Là, on s'était rendu compte que le projet était faisable et qu'au point de vue politique, on s'apercevait que c'était bon d'être à la tête de cela (...) nous, on a fait naître le projet, et un coup né, là ils nous l'ont pris, et là, ils l'ont utilisé pour faire un projet politique (...) tous les organismes pour qui c'est leur job de travailler pour le développement économique, ben là, ils avaient un beau petit moyen de démontrer qu'ils travaillaient (...) ils ont formé une corporation légale qui incorporait toutes les municipalités du Bas-Saguenay, incluant des membres telles les caisses populaires ! 
Une corporation sans but lucratif où le gouvernement devait participer au financement du projet devait être mise sur pied alors que les initiateurs du mouvement voulaient un organisme privé qui rassemblerait des promoteurs intéressés, comme cela se passe ailleurs au Québec.

Cette corporation à but non lucratif s'inscrivait maintenant dans le colimateur de la bureaucratie de l'administration publique. Aux yeux des promoteurs, ce projet devenait un dossier et ce qui paraissait facile à financer devenait au yeux des citoyens une saga tragico-comique. On leur proposait des programmes de création d'emplois « pour les aider $» . .$.

On va vous financer un étudiant pour un programme défi 25, 35 et toutes sortes de programmes gouvernementaux d'aide à l'emploi, disait-on. Alors que c'était un centre de ski qu'on voulait faire (...) À force des rincer, je me suis retrouvé presque seul avec C. D. de ma gang au C.A.

Après un an de «cheminement» en remplissage de demandes de subvention, de rencontres, de remplissage de formules, je leur ai dit, eih ! c'est pas notre vie, nous autres, des demandes de subventions (...) nous autres on était pas arrêtés par des principes politiques où il fallait passer par un avant l'autre, toute la hiérarchie politique (...) eih ! j'ai dit, ça se peut, on s'est fait embarquer raide. Mais, ils avaient le gros bout, car ils avaient réussi à avoir l'appui de la municipalité, tous les maires, les MRC, la STF, le CAA, le RAA et tout ce que tu pourras inventer comme sigles, c'était rendu qu'ils avaient 20 tiroirs de lettres d'appui (...) et là, on était en 1988, j'ai débarqué quand ils m'annoncent qu'au bout d'un an, ils venaient de décrocher un «défi 1988 ». Je suis plus capable de travailler avec vous autres. (Mais dans ma tête, je savais maintenant que je serais plus libre pour agir). Le projet du Mont-Édouard était devenu le party pour eux autres, car le dernier citoyen de l'Anse au C.A. avait été évincé par un vote de non confiance (...) Le projet aurait pu durer 20 ans (...) pour eux autres la population venait après. Pour eux autres, c'était un projet où ils pouvaient justifier leur salaire. Durant ce temps, on pouvait dire que le dossier chemine, on le structure, on l'enrobe, il est le prétexte à des réunions toutes les semaines (...) ils avaient une aide technique grâce à l'article 38 qui leur avait permis d'engager quelqu'un qui répondait à la correspondance, remplissait les demandes de subvention, etc. (...) Ça duré ainsi jusqu'au temps où je téléphone au président, Lucien Martel, pour lui dire : «Le projet du Mont-Édouard, c'est pas pour 10 ans, c'est tout de suite qu'on a besoin. Moi je vis ici et toi tu vis à Chicoutimi. Tu as un salaire de 110000 \$ /année, pour toi, ça presse pas. Icitte, les gars, c'est la valise sur le perron de la porte, sont tous prêts à partir, ils ont plus rien, sont dans la rue, plus d'emploi, sur le bien-être, sont plus capables de payer leur épicerie... et en plus, l'espoir du Mont-Édouard ne nourrit plus maintenant et là, c'est fini, vos subventions ça rentre plus (...) On était vraiment écœurés de cette partie politique (lire partie de poker)». 


\section{La révolte sociale contre la façade administrative}

\section{$\underline{\text { Retour à la table des matières }}$}

En 1989, il était devenu évident que le dossier était en train de mourir au feuilleton. Les fonctionnaires locaux arrivaient difficilement à taire les pressions des gens d'affaires de Chicoutimi et de Raymond Malenfant qui, du Valinouët et du Mont Grand Fonds, laissaient publiquement entendre que la concurrence du Mont-Édouard allait être néfaste à tout le monde.

À la suite du virage managérial effectué par l'administration publique québécoise où l'État intervient dans la sphère de l'accumulation par le truchement de «partenaires » économiques, la Corporation du Mont-Édouard devait être l'agent de promotion et le gestionnaire du projet, issu de l'initiative de la population anjeannoise. Au niveau régional, la corporation se devait de trouver des partenaires issus de tous les milieux du monde socio-économique.

«Le coût du projet totalise la somme de plus de 7 millions de dollars. Les partenaires sont actuellement : le Gouvernement du Québec (1,7 millions) ; le Gouvernement d'Ottawa (3,1 millions) ; le milieu local (667,000 \$, dont 150000 \$ venant de la Caisse Populaire, 72000 \$ des entreprises, 319,000m \$ des particuliers) ; le milieu régional (835000\$) et la Corporation du Mont-Édouard (1 million sous forme d'emprunts) $\gg^{2}$.

De même que les sommes d'argent générées peuvent être un indicateur de l'intérêt économique suscité par le projet, les intérêts économiques des groupes constitués loin de se réguler à l'intérieur d'un modèle théorique de partenariat peuvent rivaliser jusqu'à l'éclatement si l'objectif économique ne se concrétise pas rapidement. Quatre ans après le colloque de la Jeune chambre locale, le MontÉdouard était encore l'objet d'une rhétorique, tantôt éléphant blanc, tantôt chimère. Au-delà d'un État post-keynésien, le concept de partenariat subissait

2 D. Gagné et S. Rousseau, «Le partenariat et la régulation du local : le cas de l'Anse-SaintJean et Pohénogamook », U.Q.A.C., avril 1990, Maîtrise en Études régionales. 
l'insolence du jeu politique. Contre la députation régionale péquiste favorable à la réalisation rapide d'un parc recréo-touristique dans le prolongement duquel s'inscrit l'aménagement quatre saisons du Mont-Édouard, s'interposait le pouvoir libéral du gouvernement du Québec assujetti à une politique de la preuve de la rentabilité économique de tous les équipements de ski alpin avant que ne soit subventionné un nouveau projet du même type. Durant ce temps, au palier fédéral, la rhétorique du «bon garçon» attentionné cachait de plus en plus difficilement une guerre politique. Anse-Saint-Jean et le Mont-Édouard se trouvaient dans le comté voisin du Mont Grand-Fonds dont Brian Mulroney, premier ministre du Canada, est le député.

Au-delà du discours, se jouait donc une partie occulte entre tenants financiers du Valinouët qui, de Chicoutimi, cherchaient un appui tacite avec Charlevoix et, pour ce faire, jouaient la carte des statistiques de la fréquentation et le drame de la concurrence sur la mesure comptable. Et, par ailleurs, une population locale qui par ses initiatives de changer son cadre et son mode de vie, s'apercevait que la bonne volonté ne suffit pas. «La seule chose qu'un gouvernement a peur et c'est l'unique, c'est l'opinion publique », diront les leaders du comité de citoyens de l'Anse-Saint-Jean.

\section{La scie mécanique et le goupillon contre les fantômes de la société industrielle}

$\underline{\text { Retour à la table des matières }}$

Gaston Blackburn, député au Lac-Saint-Jean et ministre des Loisirs, Chasse et Pêche, déclare publiquement, peu après les élections provinciales du mois de septembre 1989, que le gouvernement du Québec n'investira plus dans les nouveaux centres de ski alpin. Pour les leaders du comité de citoyens, cette déclaration est considérée comme un soulagement car :

... là, enfin, j'ai l'occasion parfaite pour dire au monde d'arrêter d'y croire au gouvernement (...) le gouvernement venait dire exactement ce qu'il fallait dire (...) là, il venait de signer le début de la guerre, me confie l'un de mes interlocuteurs en riant encore.

Une réunion publique est immédiatement convoquée à la suite de cette déclaration. L'ensemble de la population se fusionne en un mouvement et 
l'objectif du mouvement s'incorpore en un acteur social. Toute la population se retrouve dans une communauté en action.

L'Anse-Saint-Jean était en guerre (...) Pauvres, opprimés par le gouvernement, les gens de l'extérieur s'identifiaient à notre cause, poursuit mon interlocuteur.

La montagne, qui avait fait des pionniers de l'Anse-Saint-Jean en 1838, des bûcheux de bois de pin pour l'amirauté britannique, des bûcheux de billots de 4 pieds pour les usines de pâtes et papiers, des travailleurs forestiers pour le déboisement des tracés et de l'entretien des lignes d'hydro-électricité, cette même montagne était en train de les convertir en techniciens ou en opérateurs ou en ouvriers ou encore en employés au service des équipements d'un parc récréotouristique. Pour y arriver, il fallait à nouveau reprendre la scie mécanique et les débusqueuses et ce, dans un contexte de révolte. Les instruments de travail devaient se convertir en armes et servir dans le combat pour la survie économique du village. Il fallait que, symboliquement, ce qui avait été les outils de l'exploitation forestière se change en instruments d'affirmation contre un adversaire extérieur à la municipalité qui entendait confiner l'Anse-Saint-Jean dans son rôle séculaire de village forestier organisé en fonction des papetières. Il devait être immobilisé dans le temps comme le site d'un camp de formation et de reproduction d'une main-d'oeuvre docile et bon marché qui en plus est autocontrôlé parce que vivant entre deux perspectives soit celle de la culpabilité et de l'exil intérieur (Montréal) soit celle de la honte d'être prestataires de bien-être social. Pour obtenir à nouveau des emplois, il fallait passer l'épreuve du feu. Le travail au pays de l'Anse devait être une quête aux dépens d'un adversaire aussi fort qu'occulte, aussi puissant que beau parleur. il fallait donc se réapproprier la rue, signifier au pouvoir présent, dans les appareils régionaux vus comme relais de Québec et d'Ottawa que la route traversant une section du village était la route qui conduisait désormais au Mont-Édouard et non plus une voie de transit de marchandises circulant du Haut-Saguenay (Chicoutimi-Jonquière-La Baie) à la région de Charlevoix-Côte-Nord. Il fallait afficher une nouvelle identité. En bloquant la route 170 , il y avait là symboliquement une force populaire qui se manifestait en démontrant que le vieil Anse-Sain-Jean s'était transformémétamorphosé et qu'à cela devait correspondre un nouveau rapport à l'espace, un nouvel aménagement. Il fallait, pour ce faire, arrêter aussi le temps. Il fallait arrêter le temps ancien, celui de la navette des voitures et des camions. Il n'est pas excessif de voir dans le blocage de la route par les débusqueuses, par les hommes, les femmes et les enfants et ce, par des températures qui, en ces jours de décembre 1989 , variaient entre $25^{\circ}$ et $35^{\circ}$ sous zéro, il n'est pas excessif, en effet, de voir là l'expression d'une machine décidée à imposer son chrono. Le 13 décembre 1989, le Quotidien titrait : «Gigantesque blocus à Anse-Saint-Jean » et sous la plume d'Yvon Bernier on pouvait lire : 
Un seul sujet de conversation courait sur toutes les lèvres : le projet du Mont-Édouard. Et la communauté (c'est moi qui souligne) avait tout à loisir d'en discuter, puisque les petites entreprises, les commerces, les écoles et la caisse populaire avaient fermé leur porte.

Et dans le même article relatant le blocage de route, les adversaires étaient débusqués de la façon suivante :

Les porte-parole rappellent que la population est très, très déçue du refus du gouvernement. Anse-Saint-Jean ne prise pas du tout que l'on cède aux pressions d'intérêts privés, ou pour être plus précis, que l'on prête l'oreille à l'homme d'affaires Raymond Malenfant et aux administrateurs de la corporation Le Valinouët, plutôt que de tenir compte de l'avenir de tout le Bas-Saguenay. L'article du journal conclut ainsi : «Claude Boudreault ajoute que la façon d'agir des dirigeants de la station de ski de Falardeau (le Valinouët, situé au nord de la ville de Chicoutimi) est plus que déplorable : «Ces gens respirent le même air que nous, et ils se comportent comme si la solidarité n'existait pas $»^{3}$.

En arrêtant les horloges de l'économie (les flux d'échange) et en imposant un nouveau sens à l'occupation de l'espace, Anse-Saint-Jean émettait avec clarté un nouveau message. Nous sommes les héritiers des pionniers, contre les privilèges de l'Empire britannique ${ }^{4}$ nous avons conquis la forêt et colonisé ces terres hostiles. Aujourd'hui, contre des adversaires intérieurs à qui des droits ailleurs accordés sont ici des atteintes à notre dignité, nous vous révélons debout, armes en mains, notre unanimité pour la reconquête de notre territoire. Nous ne voulons de la culture des autres. Notre idéal n'est pas celui d'être des assistés sociaux libres, à la consommation élémentaire. Conquérants hier de la forêt, conquérants aujourd'hui de la montagne.

Edgar Morin écrit : «Le temps de la vérification est nécessaire, c'est-à-dire qu'en fin de compte c'est la PRATIQUE qui donne la réponse, pratique dont les résultats sont engrangés dans le savoir collectif (la culture). Mais même alors, la pratique et la culture peuvent ne pas dissiper l'illusion (...) Mais le propre de SAPIENS est aussi la possibilité de douter de l'existence des esprits et des dieux, de séparer les mots des choses, de contester les théories qui se referment sur le monde comme si celui-ci était transparent à l'entendement. C'est-à-dire qu'il peut découvrir ce qui est indécidable en principe et ce qui est ambigu en fait, et mettre finalement en cause la vérité établie (...) le cerveau humain a besoin de l'écosystème, de la culture, de la société, de la praxis pour établir ses vérités, ce qui le pousse à chercher dans et par la nature, dans et par la culture, dans et par la société, dans et par la pratique, la solution de ses incertitudes (...) Mais à nouveau, dans le nouveau système d'idées (lire nouveau cerveau, nouvelle conscience de soi nouvelle

3 Le Quotidien, 13 décembre 1989, « Pour la réalisation du Mont-Édouard, Gigantesque Blocus à Anse-Saint-Jean ».

4 Ici, il est fait référence aux écrits de Hannah Arendt sur l'impérialisme dans son livre: Les origines du totalitarisme : L'impérialisme. Paris, Points/Fayard, 1982, 348 p. 
culture), il retrouvera l'ambiguïté et l'indécidabilité, ce qui le poussera à chercher, élaborer un méta-système » 5 .

Cette pensée d'Edgar Morin est, à la limite, contenue dans la réflexion que faisait une citoyenne d'Anse-Saint-Jean :

Je me rappelle qu'un vieux curé, en chaire, nous avait dit que lorsque la forêt ne nous nourrirait plus, ce sont vos montagnes qui le feraient.

Nouveau rapport à l'espace donc, mais nouveau rapport à la nature qui en retour construit le bagage culturel. Une culture qui structure le corps social et qui dissocie l'image onirique de l'imagination créatrice, l'homme imaginaire de l'homme imaginant, le fantasme de l'idée, l'affectivité de la praxis. L'imagination peu à peu s'incorpore dans un groupe social pour qui l'aspiration rencontre la magie ; le rêve et le poiesis touchent les mythes et leurs doubles.

Quand au mois de février 1990, les bûcherons décident de défricher « illégalement » les futures pistes de ski alpin. Quand le curé Clément Harvey, inspiré par les lectures de l'Évangile, décide dans le même temps, sans concertation, de ne plus s'alimenter, il y a là fusion de forces. La raison oblige à convenir de l'inéluctable «prédiction créatrice»: «6 millions \$ pour Parc Saguenay - OUI au Mont-Édouard » titrait le Quotidien en page couverture le 17 février 1990.

Fin du texte.

$5 \quad$ Edgar Morin, Le paradigme perdu : la nature humaine, Paris, Points, Seuil, 1979, p. 139. 